\title{
Changing Life Cycles and their Impact on Insurance
}

\author{
Didier Blanchet \\ Département des Etudes Economiques d'Ensemble, INSEE - Timbre G201, 15 Bd Gabriel Péri, \\ 92245 Malakoff Cedex, France. \\ E-mail: didier.blanchet@insee.fr
}

Demographic changes are often described as major forces that are going to shape tomorrow's economies. We examine how far such a claim is valid, shortly reviewing fields such as potential economic growth, financial markets, pensions, the demand for additional old age income insurance, health and old age invalidity. On the whole, demographics clearly matter. At the same time, its impact will be mediated by many other determinants, including changes in public policy design and behavioural responses at the individual level. The Geneva Papers (2007) 32, 1-10. doi:10.1057/palgrave.gpp.2510111

Keywords: demographics; population economics; ageing; pensions

\section{Introduction}

Demographic changes in general and the ageing process in particular are often considered one of the major challenges that developed and also developing countries will face during the next decades. These changes are expected to significantly reshape individual life cycles and their global outcomes at the macro-economic or macro-social level.

How far are such predictions reliable and what can we say about the consequences of demographic change at the meso level of a sector such as the insurance industry? This paper does not intend to present a global answer to these questions but tries to provide some basic landmarks, identifying areas where the consequences of demographic change are likely to be significant, but also mentioning areas where these consequences remain more uncertain, quantitatively or qualitatively.

We shall start by briefly recalling what are these general demographic trends and the underlying mechanisms. Although these trends are well known, there remains some ambiguity concerning the relative roles of their two main determinants, that is, mortality and fertility changes. Clarifying these roles is important for two reasons: it helps assess the reliability of projections, and the nature of ageing has to be taken into account when discussing its consequences. We shall then move on to a very simple presentation of what we know and do not know about the macro aspects of these consequences, before discussing questions more directly related to the insurance sector.

\section{Changing life cycles and population ageing: an overview}

Figure 1 gives a global view on the ageing process, both retrospective and prospective, for the last 50 years and until 2050, according to the medium variant of the last demographic projections produced by the United Nations. The indicator is the ratio of 


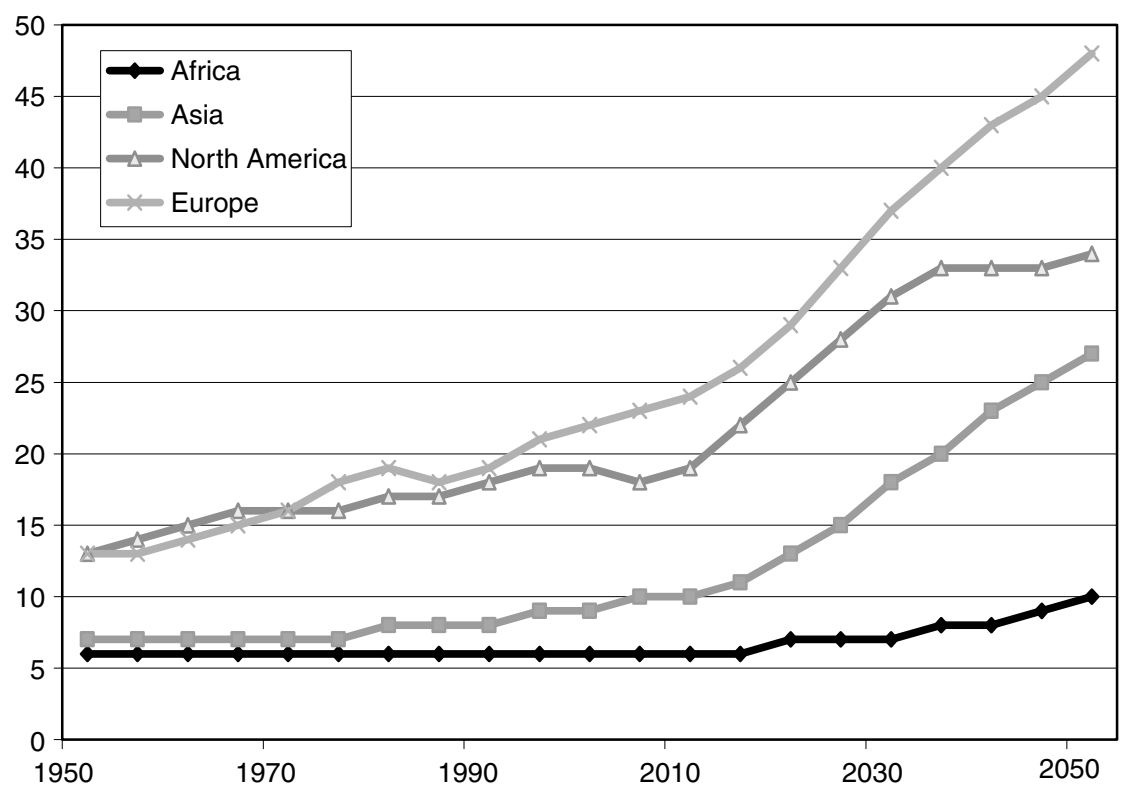

Figure 1. Ageing: a general trend (ratio $65+/ 15-64$, United Nations projections, medium variant, in per cent).

people aged 65 or plus to people in working age. To keep the graph readable, we do not give systematic evolutions for all geographic areas, but for only four of them: Europe, North America, Asia and Africa. Except for Africa, ageing is not a new phenomenon, but there is no doubt that it will significantly accelerate everywhere during the next decade. It is for European countries that the process is already the most heavily engaged and its acceleration is expected to bring the $65+/ 15-64$ ratio close to 50 per cent, meaning a still higher pensioner/worker ratio if current employment rates for the 15-65 age group were to be maintained.

What are the factors underlying such a general trend? If we concentrate on the case of a typical developed country, three factors contribute to this trend: increases in life expectancy, the baby-boom effect and a baby-bust effect.

The baby-boom effect corresponds to the fact that fertility levels were significantly higher than the replacement level until the end of 1960s. The "baby-bust" corresponds to the fact that post-baby-boom fertility levels not only returned to replacement levels but generally declined below this level, and sometimes very strongly below.

How do these factors interact? Standard demographic transition theory assumes that, from an initial stationary situation combining high mortality and high fertility, we ultimately move toward a new stationary equilibrium where life expectancy is high and where fertility has adjusted downwards to compensate for the higher survival rate of children and young adults. At the two extremes of this transition process, the age structure is fully determined by mortality, since the age structure of a stationary population exactly mirrors its survival function. Following this model, it is reasonable to consider a reference trend of "normal" ageing due to mortality change which is the 
trend that would prevail, between these two stationary states, with a permanent adjustment of fertility to current mortality conditions.

This trend actually determines the general profile of series such as the ones given in Figure 1. Around this trend, the impact of fertility levels that are above or below the current replacement rate is respectively to delay or to intensify this process of normal ageing. For instance, the baby-boom effect common to most developed countries has been, in a first step, to contain the ageing process. But such a situation could not last forever and returning to the replacement ratio not only stops the process, it also forces the return to the "path" of normal ageing. If ageing is measured by the ratio of $65+$ to 15-64, this occurs when the first cohorts of baby-boomers reach age 65, and this corresponds to the inflexion that appeared in Figure 1, that is, what is sometimes depicted as a "papy-boom" effect.

Concerning the "baby-bust", its effect consists in an aggravation of ageing beyond its normal trend. This aggravation will itself be permanent or only temporary depending on future evolutions of fertility. This baby-bust effect already plays a significant role in countries where fertility has dropped to very low levels. The effect is more a very long term one in countries, like France, where the drop in fertility has not been that large.

This presentation helps us sort out what is unavoidable or avoidable, certain or uncertain in this ageing process. The "normal ageing" and "papy-boom" effects are largely unavoidable: there is a great deal of irresponsibility and lack of realism in pretending that such effects could be counterbalanced by a new surge in fertility levels, or by increasing reliance on migration from developing countries. Migration can play a positive role, but is certainly unable to fully avoid the consequences of increased longevity.

The main uncertainties therefore concern the question of knowing how long and how deep the baby-bust effect is going to be and the way "normal ageing" will be affected by future evolutions of mortality. On this latter question, there are two opposite schools of thought:

- One position is rather optimistic. It derives from the fact that past demographic projections have been generally relatively conservative on mortality. This leads some specialists to predict that life expectancy will go on increasing at a sustained rate.

- But some others underline factors going in the opposite direction: decreasing returns on medical progress, new pathologies, delayed impact of a decreasing selection at birth. ${ }^{1}$

At this stage, the only thing that we can say is that we do not find any apparent inflexion for western developed countries (Figure 2): things remain opened. But this uncertainty should not be a motive for inaction. It is rather a point in favour of flexible systems able to adjust to unexpected changes in this mortality trend: systems indexing age at retirement on life expectancy are one good example for that.

\footnotetext{
${ }^{1}$ e.g. Olshansky et al. (2005).
} 


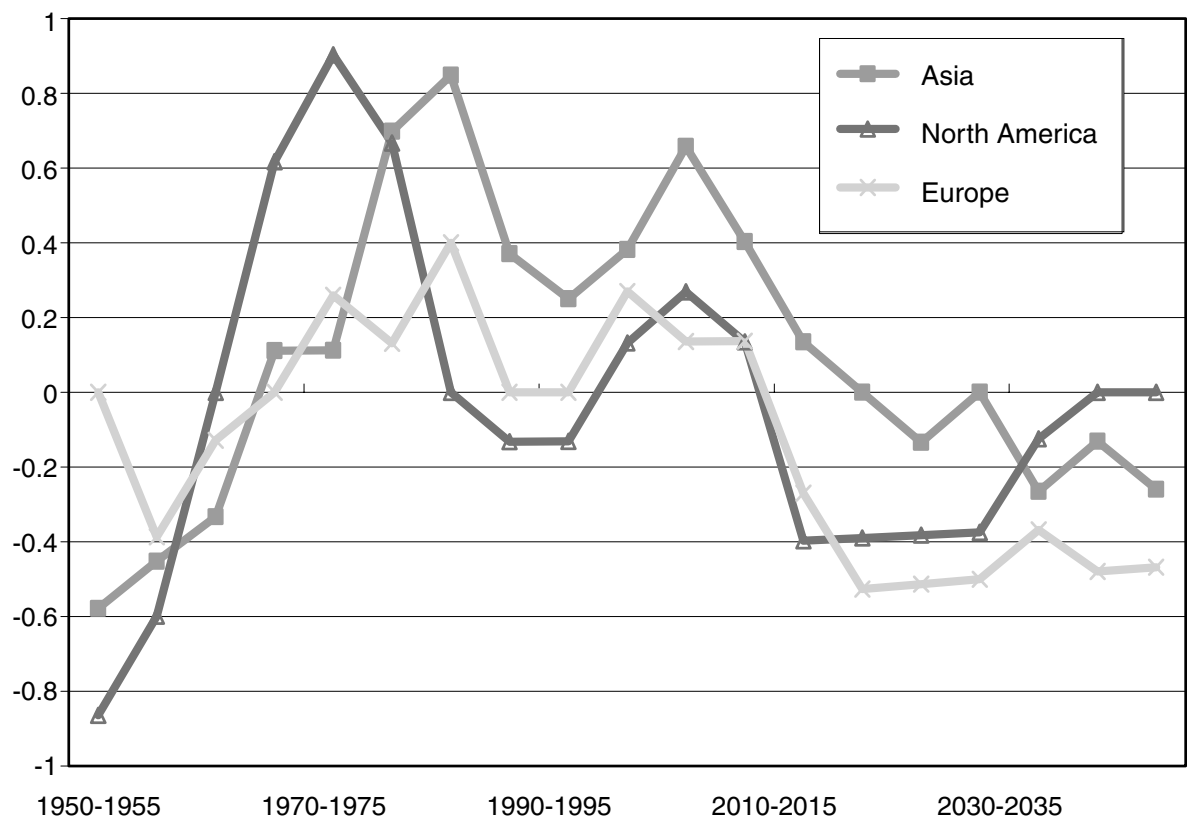

Figure 2. Contribution of the global dependency ratio to growth of GDP/head (United Nations projections, medium variant, in per cent, + personal computations).

\section{About macroeconomic consequences: the impact on global economic growth}

Whatever this uncertainty on the intensity of ageing, the qualitative trend is warranted: a progressive slowdown of global demographic growth associated with an increasing ratio of inactive to active people. What can be said of the macroeconomic consequences of such changes?

Assessing general consequences of population change on economic growth or well being is a question that is approximately as old as economic science itself, and to which we still do not have a clear answer. Economists or demographers should not be blamed for this lack of general answer. The ambiguity is a real one, not the result of insufficient empirical or theoretical investigations. Population change can indeed produce very different results depending on a variety of contextual elements: institutions, availability of natural resources, capacity to transform the productive potential of additional people into actual production. Contrary to the reasonable amount of confidence that we could have on the reliability of demographic projections, uncertainty now predominates, even from a qualitative point of view. Are returns to population size or growth positive or negative, as argued respectively by populationist or Malthusian currents? The answer is hard to give, and empirical evidence does not help us a lot on this point.

In this context, one first step can be to go back to the simplest of first-order relations. The standard view about potential growth is that it is the sum of the growth 
rate for active population and a coefficient of technical progress. Let us assume that the latter one is more or less orthogonal to demographic change. Then we get a one for one relationship between labour force growth and the growth rate of total output. This leads to a first order effect of demographic change on the growth rate of income per capita equal to the difference between the growth rate for the active population and total labour force, that is, the rate of change for the dependency rate.

The computation of this effect is simple and leads to effects that are not trivial. An illustration is given by Figure 2. For Europe, this ceteris paribus contribution of demographic change to the growth of GDP/capita should soon become negative, representing a yearly loss of about 0.5 per cent in output per head each year over the next $30-40$ years. This is not to the point where economic growth itself would be jeopardized. We can still believe in the possibility that productivity change will be higher than this 0.5 per cent in absolute terms. But the impact remains nevertheless significant.

Can we imagine second-order effects that could either offset or reinforce this impact of demographic change on economic growth? We mentioned uncertainty about the importance of effects of increasing or decreasing returns. Even if theories of endogenous technological change have renewed interest for the former category, empirical evidence remains too weak to incorporate them in our projections. In fact, it is likely that externalities that are susceptible to boost growth in modern and integrated economies are no longer that much dependant on population size or density and have more to do with agglomeration effects, ability to attract or retain very high qualified workers or the structure of labour markets.

One more direct link between ageing and average productivity could eventually come from the fact that productivity varies with age. On this point, however, ex ante simulations show that the interaction between realistic age profiles for productivity and ageing patterns does not result in large changes in productivity. ${ }^{2}$ This result is more general than that and applies to many examples of interactions between the agespecific profile of a variable at the micro level and changes in the global age structure of the population. For variables whose link with age is relatively smooth, the interaction at the macro level between this age effect and changes in age structure very often leads to very small effects.

On the whole, the prudent approach considering that the dominant ceteris paribus effect of demographic change on economic growth was the one depicted by Figure 2 is not without foundations.

\section{Beyond effects on total output: impacts on the repartition of incomes and wealth}

A given effect on total output can come along with large effects on the repartition of this output. If we believe in the idea that prices and relative scarcity are correlated, then a simple idea is that demographic slowdown, by increasing the relative scarcity of labour compared to capital, should depress the value or remuneration of the latter

\footnotetext{
${ }^{2}$ Blanchet (1993).
} 
one. One elaborated version of this simple view is of particular interest to all actors in capital markets, including insurers and has come to be known as the asset meltdown hypothesis $(\mathrm{AMH})$. It not only derives from the natural dynamics of the capital/labour ratio in front of a declining population, it also incorporates elements from basic life cycle theory (LCT), according to which active households are net savers on the average, because they are in a phase of preparation for retirement, while older households are dissavers because they consume part of their capital to complete the retirement income they get from their pension systems.

According to the $\mathrm{AMH}$, there is a strong potential interaction between LCT behaviour and the baby-boom/baby-bust effects that have been described above. In a first step, baby-boomers who are still in activity save intensively to prefinance a retirement period which is expected to be long, and for which they expect low replacement rates. Capital markets therefore face excess demand, and capital prices go up. Once these baby-boomers are in their retirement period, the situation reverses. Capital markets move to excess supply, and assets that have been bought at high prices must be resold at depressed ones.

How relevant is such a thesis? There is at least one useful element in the AMH: it reminds us that, in principle, the relative performance of funded pension systems is not less affected by demographic changes than the performance of unfunded ones. This is true even if we believe in the idea that funded systems have higher returns in the long run: in that case, returns on PAYG contributions and on savings respond to demographic shocks with parallel moves around different long-run levels.

But this does not lead us to the point where the AMH can be used to predict a dramatic decline in asset prices for the next decades. From an empirical point of view, evidence in favour of a strong impact is difficult to find, ${ }^{3}$ due to the lack of past similar demographic shocks that could serve as points of reference. The AMH must also be relativized due to the fragility of its premises. In particular, the LCT representation of savings behaviour remains challenged on many grounds. Agents are more myopic and less rational than assumed by this theory. Under current conditions, we also know that people remain savers during their retirement period, due to the importance of the bequest motive.

Maybe individual savings behaviour will come closer to LCT in the future if pension levels decline and if institutional innovations make it easier to consume one's capital after retirement (e.g., reverse mortgage). But another key variable in that case is the future level of asset demand in front of this increased supply by old life-cyclers. If the following cohorts of workers have the same need of preparing for their retirement, then this additional supply of capital should meet a demand that will itself remain dynamic. The risk of a strong setback in asset prices appears only if these new cohorts are much less numerous than the cohorts of their parents, but this would be essentially true for closed countries with large baby-bust effects. It does not apply to the worldwide level of integrated financial markets where these baby-bust effects are heavily diluted.

\footnotetext{
${ }^{3}$ Poterba (2004).
} 
To conclude on this point, despite the fact that the AMH hypothesis remains partly relevant and deserves further examination, its impact should not be overestimated. Many other sources of fluctuations of asset values have existed and will go on existing in the future. In particular, it would be excessive to argue that demographic changes are responsible for sudden fluctuations that affect these markets. Or, if they do, it is only in a very indirect way, by feeding irrational but self-fulfilling beliefs that explain the volatility of these markets. In that case, the problem has more to do with collective representations of consequences of demographic change than with demographic changes themselves.

\section{Some more specific consequences for insurance: the demand for annuities}

Let us turn to real consequences of demographic changes that have a direct impact on the demand for insurance. All aspects of the demand for insurance are likely to be affected by population ageing but in many instances, the impact, once again, must not be overestimated. In many instances, the impact can even be very weak or at least much less important than the impact of other determinants. The point is the same than the one that has been raised concerning the impact of demographic changes on average productivity. As long as the phenomenon of interest only has a moderate and relatively smooth link with age at the individual level, demographic change only has a tiny effect on the global measure of this phenomenon at the macro level.

This must lead us to concentrate our attention on subdomains where the relationship with age is the most pronounced. Without surprise, these domains are those connected to old age: pensions, health, and old age invalidity.

Some aspects of the pension issue have been already mentioned when discussing the $\mathrm{AMH}$. The question which is of interest for insurers is the combined impact of ageing and pension reform on the demand for additional old age income insurance. Most countries have already implemented significant pension reforms that have generally paved the way for relatively large reductions of pension entitlements. But the impact on the demand for compensating annuities that could be supplied by the insurance sector will depend on the way these reductions will take place.

If they essentially come from the fact that reforms succeed in maintaining older workers in the labour market and in stabilizing the length of the retirement period but without any strong negative impact on replacement levels, then the need for additional coverage would remain weak.

Another scenario would be the one where reforms are successful in controlling the length of the retirement period but without any equivalent success in raising effective employment rates for older workers, in which case a specific need for coverage of the pre-retirement period may develop. This fear is particularly strong in France where actions have been taken to raise retirement ages in a context where demand for older workers by firms remains structurally weak. In that case, the demand for complementary coverage would rather concern the phase of transition between the last employment and retirement stricto sensu. But are private markets ready to cover a risk that is nothing else than a subcategory of unemployment risk, a field in which, for various reasons, private coverage is hard to implement? 
If the reduction of entitlements is rather the result of lower replacement levels, then the need for additional coverage by non-myopic agents should increase strongly, and we turn back to a field where there is more potential for market solutions, but once again with some limits. In particular, one aspect of the expected decrease of pensions compared to average wages is the adoption of less generous indexation rules for pensions during retirement. If the issue is this one it essentially applies to the "oldest olds" and the problem is here to provide instruments offering a well-indexed coverage in the long run. It is not sure that market instruments are at their comparative advantage in front of this specific problem.

\section{Health expenditures and the oldest olds}

The oldest olds are also the ones who should be the most intensively concerned by increasing demand for health services and services required to deal with old age invalidity. Before detailing this problem, let us come back shortly to the general impact of demographic change on health expenditures.

Invoking demographic factors as one of the causes of increasing health expenditures is frequent. Such an assertion is sometimes used to deny the opportunity of regulating these expenditures. The argument is that if their increase is the normal result of demographic trends, trying to slow down this trend is not only difficult but unfair since it would mean a reduction in the coverage of individual needs.

This simple view is not supported by empirical evidence, at least for the past. As far as past trends are concerned, the role of demographic factors has been very small. The growth of expenditures essentially resulted from their increase at all ages, not from increasing numbers in age groups for which expenditures are the highest. This growth observed for each age group itself resulted from income effects, technological changes (which increase the spectrum of efficient but costly treatments that are potentially available for given pathologies), lack of regulation for both supply and demand, or eventually some sociological effects, that is, the fact that cohorts of people who have been accustomed to low consumption levels have been progressively replaced by cohorts of people who have been accustomed to strongly rely on the health system. It is important to insist upon the fact that this latter effect should not be considered as an impact of population ageing: it is a cohort effect that would occur even in a perfectly stationary demographic environment.

How will the relative shares of these different factors evolve in the future? There is little doubt that the role of the purely demographic factor should increase, since ageing will itself accelerate. But simple calculations show that this acceleration should have a smaller impact on health expenditures than the one that, before reforms, could be foreseen for pensions. The reason for this difference is that the relation of expenditures to age is much more progressive for health expenditures than for pension expenditures. Demographic changes will only remain one among the many factors pushing health expenditures upwards.

Even for the sub-domain of health-related expenditures that is potentially the most sensitive to ageing, that is, expenditures related to old age invalidity, some uncertainty exists, which reflects the uncertainty concerning what will be the future length of this 


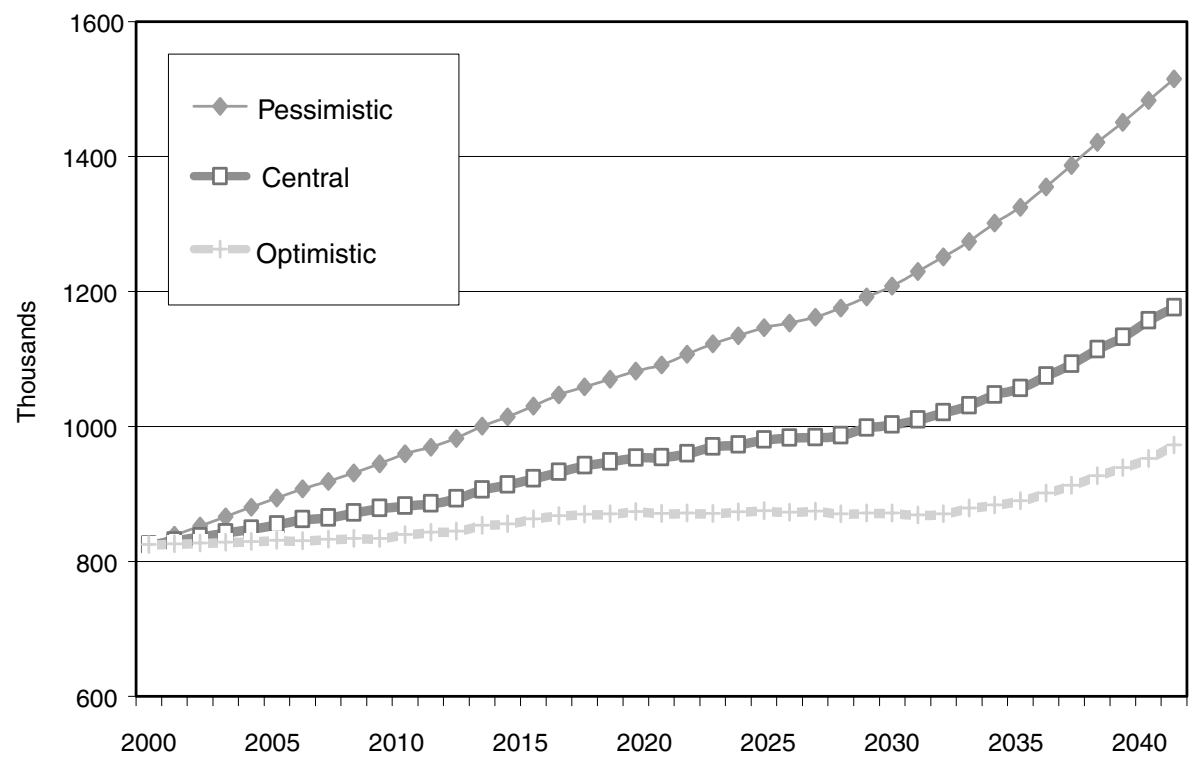

Figure 3. Projection of old age incapacity in France according to three demographic scenarios, France. Source: Duée and Rebillard, 2004.

period of invalidity which often takes place before death. Figure 3 gives one French illustration of this phenomenon with projections based on a microsimulation model where three assumptions are retained. In the medium scenario, the length of this period spent in invalidity is assumed to be stable, at approximately 1.4 years for men and 2.5 years for women. In the pessimistic case, it is supposed to increase up to respectively 1.8 and 3.2 years, and to decrease to 1.2 and 2.2 years in the optimistic case.

In all cases, the number of people in old age invalidity is expected to increase, but with a considerable margin of uncertainty. One can also note that the major point of inflexion for all three scenarios is in a future that remains relatively remote. It corresponds to the period where first cohorts of baby-boomers will enter into ages of high prevalence, and this takes place about 25 years after their entry into retirement, at an age of about 85 on average.

Here again, the magnitude of the induced demand for private coverage of this risk will depend on institutional arrangements concerning basic coverage. But the ability of private markets to provide this additional coverage is itself affected by this uncertainty concerning the macro-prevalence of this risk.

\section{Some concluding remarks}

Commenting about consequences of demographic change often looks like commenting about a glass that is halfway full or half way empty. The qualitative orientation of changes is relatively certain. Up to a certain point, we can also give reasonable 
10

intervals of confidence for the quantitative importance of ageing. But consequences of these changes at the macro or meso level raise more questions. In many instances, they do not seem likely to have the huge consequences that are often feared. But they are not marginal either. They just need to be placed in their right place, that is one among the many forces that are going to shape tomorrow's economy.

\section{References}

Blanchet, D. (1993) 'Does an aging labour force call for large adjustments in training or wage policies?', in P. Johnson and K.F. Zimmermann (eds.) Labour Markets in an Ageing Europe, Cambridge: CEPR/Cambridge University Press, pp. 126-143.

Duée, M. and Rebillard, C. (2004) La dépendance des personnes âgées: une projection à long terme, Working Paper INSEE/DESE G2004-03.

Olshansky, S.J., Passaro, D.J., Hershow, R.C., Layden, J., Carnes, B.A., Brody, J.D., Hayflick, L., Butler, R.N., Allison, D.B. and Ludwig, D.S. (2005) 'A potential decline in life expectancy in the United States in the 21st century', The New England Journal of Medicine 352(11): 1138-1145.

Poterba, J.M. (2004) The impact of population aging on financial markets, NBER Working Paper No. W10851.

\section{About the Author}

Didier Blanchet has worked as researcher in the French national institute of demographic studies (INED) until 1993. Since then, he has occupied various positions within the French national statistical institute (INSEE) where he is currently head of the department of general economic studies. 